\title{
Facile Synthesis of 1,2,3,4-Tetrasubstituted Pyrroles from Baylis-Hillman Adducts
}

\author{
Scong Jin Kim, Hoo Sook Kim, Tack Hycon Kim, ${ }^{\dagger}$ and Jae Nyoung Kim ${ }^{*}$ \\ Department of Chemisty and Institute of Basic Science, Chonnam National University, Gwanght 500-757, Korea \\ E-mail: kiminía chonnam.ackr \\ "Department of Applied Chemistry, Chonnam National Linersity, Gwangin 500-757, Korea \\ Recened June 4, 2007
}

Key Words : Pyrroles, Baylis-Hillman adducts, PCC, Decyanomethylation

Suitably functionalized pyrroles are the basic skeleton of many biologically important substances and numerous synthetic methods of pyrroles have been investigated extensively. ${ }^{12}$ However, the synthesis of pyrrole derivatives from Baylis-Hillman adducts was not developed much. ${ }^{2}$ Recently, we reported the synthesis of 2,3,4-trisubstituted pyrroles starting from the rearranged $a z a$-Baylis-Hillman adducts (Scheme I).

Meantime we presumed that we could synthesize 1,2,3,4tetrasubstituted pyrrole derivatives by using the synthetic approach in Scheme 2. As shown in Scheme 2, we imagined that the reaction of Baylis-Hillman acetate 1 , as the representative example, and secondary amine derivatives $\mathbf{2 a - d}$ could give the corresponding $S_{.} 2^{\prime}$ product $\mathbf{3 a - d}$, which could be cyclized to 4a-d under basic conditions. The following acid-catalyzed dehydration and concomitant double bond isomerization of $\mathbf{4 a - d}$ would provide desired pyrroles 5a-d.

Among the examined conditions the use of $\mathrm{K}_{2} \mathrm{CO}_{3}$ in $\mathrm{CH}_{3} \mathrm{CN}$ gave the best results for the preparation of 4a-d. As expected we could not observe the formation of 3 (except for 3c, entry 3 in Table 1), instead we obtained $\mathbf{4 a - d}$ directly in $50-74 \%$ yields as inseparable sin/ani mixtures in a one-pot reaction. Based on the ${ }^{1} \mathrm{H}$ NMR spectra of $\mathbf{4 a - d}$ the ratio of syn/ani was $4: 1$ to $2: 1$ (footnotes b-d in Table I), however, we did not confirm which isomer is the major one. For the reaction of 1 and $2 \boldsymbol{c}$ we isolated $3 \mathbf{c}$ in $34 \%$ yield (entry 3 in Table 1) together with $\mathbf{4 c}$ in $50 \%$ yield. For the synthesis of compound $\mathbf{4 d}$ (entry 4) we used $\mathbf{2 \mathbf { d } ^ { 5 }}$ in slightly excess amount (footnote $\mathrm{e}$ in Table 1). The following dehydration step of 4a-d was carried out under the influence of $p$-TsOH $(20-40 \mathrm{~mol} \%)$ in benzene and we obtained the desired compounds 5a-d in 41-64\% yields. Isomerization of double bond occurted during the dehydration stage simultaneously to afford pyrroles directly. The results are summarized in Table 1.

However, the reaction of $\mathbf{1}$ and $\mathbf{2 e}$ showed somewhat different reactivity as compared with those of 2a-d (Scheme 3 ). When we cartied out the reaction of 1 and $2 \mathrm{e}$ in $\mathrm{CH}_{3} \mathrm{CN}$ at room temperature the reaction did not show the formation of any new compounds in appreciable amounts presumably due to the limited solubility of $2 \mathrm{e}$ in $\mathrm{CH}_{3} \mathrm{CN}$. Thus we elevated the temperature to refluxing, however, rearranged acetate was the major product in this case. After many trials we could obtain $3 \mathbf{e}$ in $74 \%$ yield in aqueous $\mathrm{CH}_{3} \mathrm{CN}$ at room temperature. In aqueous $\mathrm{CH}_{3} \mathrm{CN}$ the compound $2 \mathrm{e}$ was dissolved completely and the reartangement of acetate group of 1 to the primary position was minimized at room temperature. With this compound $3 e$ in our hand we prepared $4 \mathrm{e}$ under the same conditions of Table $1\left(\mathrm{CH}_{3} \mathrm{CN}\right.$,

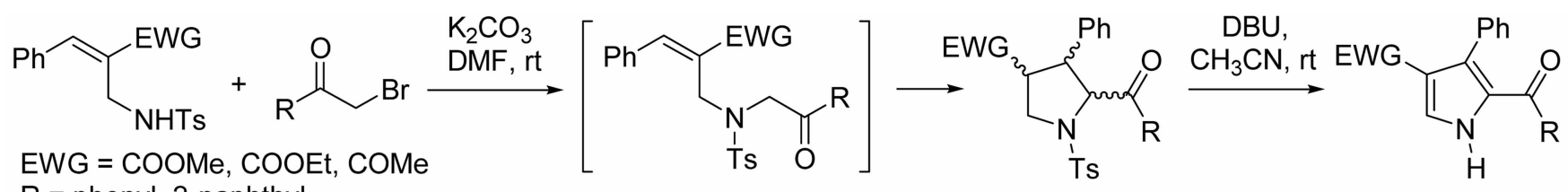
$\mathrm{R}=$ phenyl, 2-naphthyl

Scheme 1

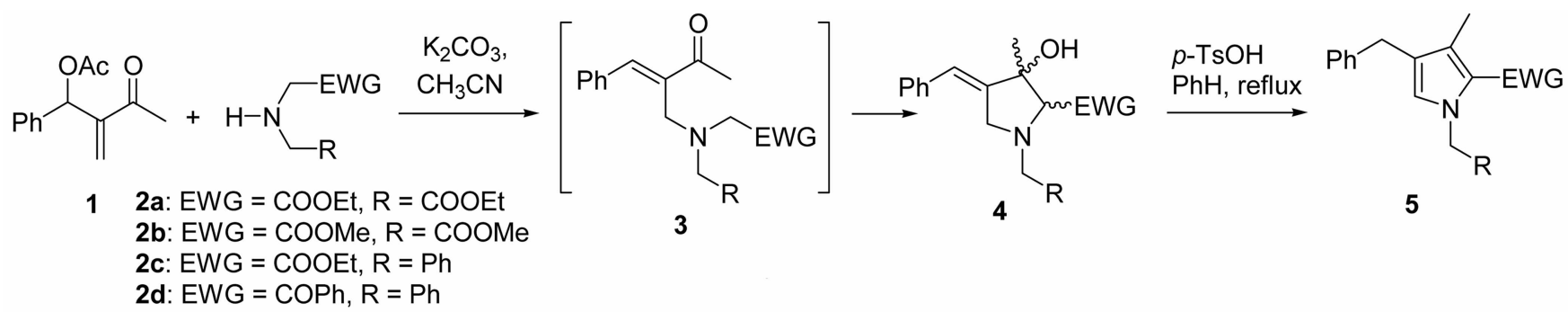


Table 1. Synthesis of 1,2,3,4-tetrasubstituted pyrroles

\begin{tabular}{|c|c|c|c|c|c|}
\hline Fintry & $1+2$ & Conditions & $3(\%) 4(\%)$ & Conditions & $5(\%)^{t}$ \\
\hline I & $1+2 a$ & $\begin{array}{l}\mathrm{K}_{3} \mathrm{CO}_{3} \text { (l.I equiv) } \\
\mathrm{CH}_{3} \mathrm{C} . \mathrm{V} . \text { retlux. } 27 \mathrm{~h}\end{array}$ & 3a (nd) $)^{s} /$ a $(69)^{b}$ & $\begin{array}{c}p \text {-TsOH (20 mol\%) } \\
\text { PhH. retlux. } 10 \mathrm{~h}\end{array}$ & 5a $(64)$ \\
\hline 2 & $1+2 b$ & $\begin{array}{l}\mathrm{K}_{2} \mathrm{COO}_{3} \text { (l.I equiv) } \\
\mathrm{CH}_{3} \mathrm{CN}_{2} \text { rellux. } 26 \mathrm{~h}\end{array}$ & $\mathbf{3 b}(\mathrm{nd})^{t} / \mathbf{4 b}(71)^{c}$ & $\begin{array}{c}p \text {-TsOH (20 mol\%) } \\
\text { Phl I. rellux. } 12 \mathrm{~h}\end{array}$ & Sb $(47)$ \\
\hline 3 & $1+2 c$ & $\begin{array}{c}\mathrm{K}_{2} \mathrm{CO}_{3}(2,2 \text { equiv) } \\
\mathrm{Cl}_{3} \mathrm{CN} \text {. rellux, } 7 \text { days }\end{array}$ & $3 \mathrm{c}(34) / 4 \mathrm{c}(50)^{f t}$ & $\begin{array}{l}\text { p-Tsol i (40 mol\%) } \\
\text { Phll, reflux. } 2 \text { days }\end{array}$ & $5 c(56)$ \\
\hline 4 & $1-2 d^{\prime}$ & $\begin{array}{c}\mathrm{K}_{2}\left(\mathrm{CO}_{3}(\mathrm{I}, \mathrm{I} \text { equiv })\right. \\
\mathrm{CH}_{3} \mathrm{CN}, \mathrm{rt} . \mathrm{Ih}\end{array}$ & 3d $(\mathbf{n d})^{d} / 4 \mathbf{d}(74)^{d}$ & $\begin{array}{l}\text { p-Tsol l (20 mol\%) } \\
\text { Phl I. rellux. } 12 \mathrm{~h}\end{array}$ & $5 d(4 I)$ \\
\hline
\end{tabular}

"Xd means not detected. "The ratio is 2:1 (swn anti mixture). "The ratio is $4: 1$ (slm anti misture). "The ratio is $3: 1$ (swn anti mixture). "Starting material $2 \mathbf{d}$ was prepared by the reaction of hemzylanine and phenacyl bronide according to the relerence." Ihe cornpound $2 \mathbf{d}$ was unstable thus we used this compound in a crude state and we used 0.91 cquiv of 1 . Isolated yicld.<smiles>N#CCNCC#N</smiles>

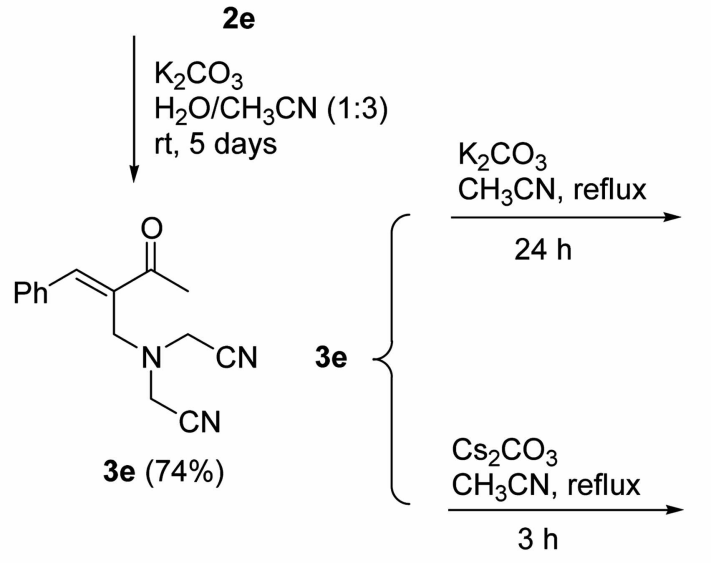

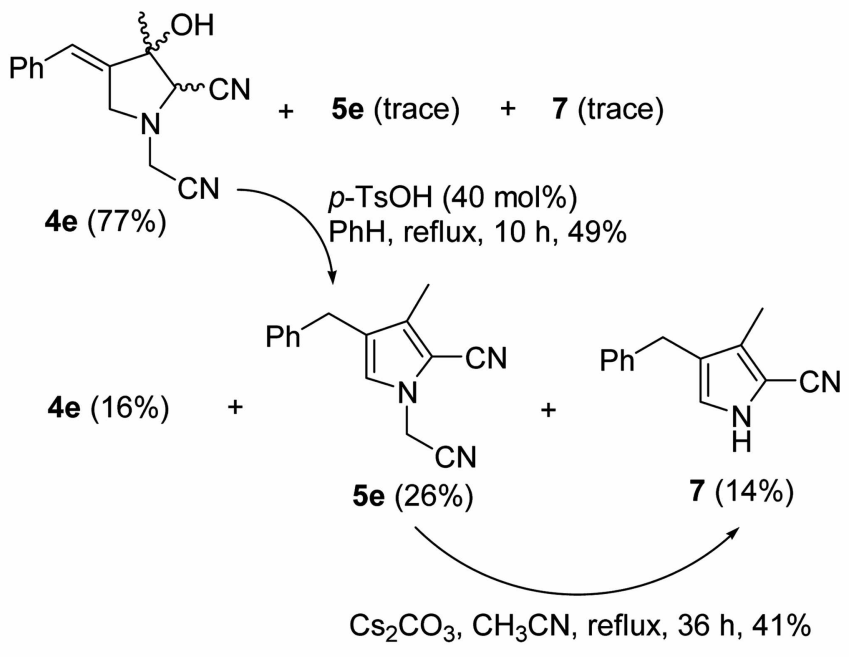

Scheme 3

$\mathrm{K}_{2} \mathrm{CO}_{3}$, reflux, $24 \mathrm{~h}$ ) in $77 \%$ yield (syn/anti, $3: 2$ ). Dehydration of $4 \mathbf{e}$ under the same conditions $(p-$ ']sOH/benzene reflux) afforded $\mathbf{5 e}$ in $49 \%$ yield. During the synthesis of $\mathbf{4 e}$ we observed the formation of trace amounts of $5 \boldsymbol{e}$ and 7 . It is interesting to note that the yields of $5 e$ and 7 were increased with concomitant decrease of $4 \mathbf{e}$ when we used $\mathrm{Cs}_{2} \mathrm{CO}_{3}$ $\left(\mathrm{CH}_{3} \mathrm{CN}\right.$, reflux, $\left.3 \mathrm{~h}\right)$. The formation of pyrrole derivative 7 can be explained by decyanomethylation of $5 \mathbf{e}^{6}$ and we confirmed the conversion experimentally by transforming 5 e into 7 under the same conditions $(41 \%$ and recovered $5 \mathbf{e}$ in $10 \%$ ).

Finally, we examined the possibility for the oxidation of $5 a$ into 4-benzoylpyrrole derivative $\mathbf{6}$ as in our previous oxidation involving PCC (pyridinium chlorochromate) in a similar syatem. ${ }^{7}$ However, the yield of oxidized compound $\mathbf{6}$ was very low to be useful in a synthetic point of view. It is interesting to note that the oxidation with the precursor 4 a instead of 5a showed somewhat improved yield.

In summary, we disclosed the synthesis of poly-substituted

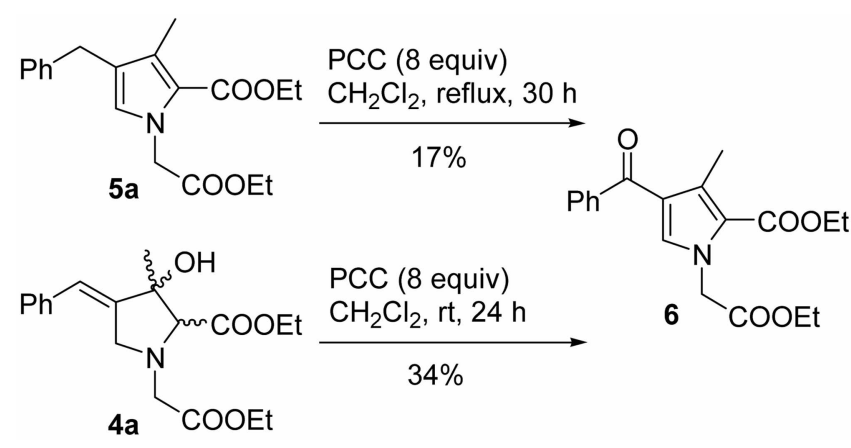

Scheme 4

pyrrole derivatives from the reaction of Baylis-Hillman acetate and some secondary amine compounds. ${ }^{8}$

\section{Experimental Section}

Typical experimental procedure for the synthesis of compounds $4 a$ and $5 a$, and the spectroscopic data of $3 c$, 
3e, ta-e, 5a-e, 6, and 7 are as follows. A stirred mixture of 1 (218 mig. $1.0 \mathrm{mmol}), 2 \mathrm{a}$ ( $189 \mathrm{mg} .1 .0 \mathrm{mmol}$ ). and $\mathrm{K}_{2} \mathrm{CO}_{3}$ (152 mg. $1.1 \mathrm{mmol}$ ) in $\mathrm{CH}_{3} \mathrm{CN}(5 \mathrm{~mL})$ was heated to reflux for $27 \mathrm{~h}$. After the usual aqueous workup procedure and column chromatographic purification process (hexanes/ EtOAc. 3:1) we obtained ta as colorless oil. $240 \mathrm{mtg}(69 \%)$. A solution of $4 \mathrm{a}(174 \mathrm{mg}, 0.5 \mathrm{mmol})$ and $p-\mathrm{TsOH}(19 \mathrm{mg}$. $0.1 \mathrm{mmol}$ ) in benzene (4 mL) was heated to reflux for $10 \mathrm{~h}$. After the usual aqueous workup procedure and columm chromatographic purification process (hexanes/EtOAc. 6:1) we obtained $5 \mathrm{a}$ as a white solid. $105 \mathrm{mig}(64 \%)$.

Compound 3c: 34\%; colorless oil: IR (film) 2924. 1737. 1666. $1231,1189.1029 \mathrm{~cm}^{-1}$ : ${ }^{1} \mathrm{H}$ NMR $\left(\mathrm{CDCl}_{3}, 300 \mathrm{MHz}\right) \delta$ $1.19(\mathrm{t}, J=7.2 \mathrm{~Hz}, 3 \mathrm{H}), 2.42(\mathrm{~s}, 3 \mathrm{H}), 3.22(\mathrm{~s} .2 \mathrm{H}) .3 .76(\mathrm{~s}$. $2 \mathrm{H}), 3.81$ (s. $2 \mathrm{H}) .4 .0+$ (q. $J=7.2 \mathrm{~Hz}, 2 \mathrm{H}), 7.19-7.27$ (m. $5 \mathrm{H}), 7.32-7.42(\mathrm{~m} .3 \mathrm{H}), 7.55-7.58$ (n. $3 \mathrm{H}) ;{ }^{13} \mathrm{C}$ NMR $\left(\mathrm{CDCl}_{3}, 75 \mathrm{MHz}\right) \delta 14.14,26.70,49.21,53.37 .57 .85,60.07$. $127.10,128.18$. 128.37, 128.83. 129.11. 130.05, 135.11. 138.59. 139.04, 141.62. 171.18. 200.85.

Compound 3e: $74 \%$; colorless oil: IR (film) 2246. 1664 . $142 \mathrm{l}, 1230.1132 \mathrm{~cm}^{-1}:{ }^{1} \mathrm{H} N \mathrm{NR}\left(\mathrm{CDCl}_{3} .300 \mathrm{MHz}\right) \delta 2.51$ (s, $3 \mathrm{H}), 3.55$ (s. $4 \mathrm{H}) .3 .64(\mathrm{~s}, 2 \mathrm{H}) .7 .42-7.49(\mathrm{~m}, 5 \mathrm{H}) .7 .85(\mathrm{~s}, 1 \mathrm{H})$.

Compound ta: $69 \%$ (sy chti, 2:1): colorless oil: IR (film) 3446. 2981, 1738. 1448. 1195, $1097 \mathrm{~cm}^{-1}$ : ${ }^{1} \mathrm{H} \mathrm{NMR}$ (CDCl . $300 \mathrm{MHz}$, major isomer) $\delta \mathrm{l} .27(\mathrm{t} . J=7.2 \mathrm{~Hz}, 3 \mathrm{H}) .1 .31$ (t. $J$ $=7.2 \mathrm{~Hz}, 3 \mathrm{H}$ ). $1.64(\mathrm{~s}, 3 \mathrm{H}) .2 .80$ (br s, IH). $3.51-3.84(\mathrm{ml}$. $4 \mathrm{H}), 4.11-4.36(\mathrm{~m} .5 \mathrm{H}) .6 .6 \mathrm{l}$ (t. $J=2.4 \mathrm{~Hz} . \mathrm{lH}) .7 .20-7.24$ (m, 3H). 7.28-7.36 (n, $2 \mathrm{H})$.

Compound $+\mathbf{b}: 71 \%$ (sm anti, 4:1); colorless oil: IR (film) 3452. 2954. 1747. 1693. 1442. 1213. $1178 \mathrm{~cm}^{-1}$; ${ }^{1} \mathrm{H}$ NMR $\left(\mathrm{CDCl}_{3}, 300 \mathrm{MHz}\right.$. major isomer) $\delta 1.63(\mathrm{~s}, 3 \mathrm{H}) .3 .51-3.90$ $(\mathrm{m}, 6 \mathrm{H}), 3.70(\mathrm{~s}, 3 \mathrm{H}) .3 .77(\mathrm{~s}, 3 \mathrm{H}) .6 .6 \mathrm{l}(\mathrm{t}, J=2.4 \mathrm{~Hz}, \mathrm{IH})$. $7.20-7.26$ (n. $3 \mathrm{H}), 7.27-7.36(\mathrm{~m}, 2 \mathrm{H})$.

Compound 4c: $50 \%$ (stn imti. $3: 1)$; colorless oil; IR (film) 3454. 2981, 1739. 1448. 1261, $1196 \mathrm{~cm}^{-1}:{ }^{1} \mathrm{H} \mathrm{NMR}\left(\mathrm{CDCl}_{3}\right.$. $300 \mathrm{MHz}$, major isomer) $\delta 1.32$ (t. $J=7.5 \mathrm{~Hz}, 3 \mathrm{H}$ ). 1.60 (s. $3 \mathrm{H}$ ), 2.75 (br s. IH). 3.34-3.65 (m. 3H). 3.94-4.05 (m. $2 \mathrm{H}$ ). $4.21-4.31$ (m. 2H). 6.56 (t. $J=2.4 \mathrm{~Hz}, 1 \mathrm{H}), 7.15-7.21$ (m. $3 \mathrm{H}) .7 .24-7.39(\mathrm{~m}, 2 \mathrm{H})$

Compound $4 \mathrm{~d}: 74 \%$ (sn anti, $3: 1$ ): colorless oil: IR (film) 3438, 1676, 1448, 1228, $1180,1092 \mathrm{~cm}^{-1}$ : ${ }^{1} \mathrm{H} \mathrm{NMR}$ (CDCl${ }_{3}$. $300 \mathrm{MHz}$, major isomer) $\delta 1.55$ (s. 3H). 2.68 (br s. IH). $3.38-4.23$ (m. $4 \mathrm{H}) .4 .38(\mathrm{~s}, \mathrm{IH}), 6.53(\mathrm{t}, J=2.4 \mathrm{~Hz}, \mathrm{IH})$. 7.17-7.34 (m. 10H) .7.43-7.49 (m, 2H). 7.54-7.60 (m, 1H). $7.93-7.97(\mathrm{~m}, 2 \mathrm{H})$

Compound 4e: $77 \%$ (sin anti. 3:2); colorless oil: IR (film) 3429. 2925, 2222, 1448, 1261, $1101 \mathrm{~cm}^{-1}$ : ${ }^{1} \mathrm{H} \mathrm{NMR}\left(\mathrm{CDCl}_{\hat{j}}\right.$. $300 \mathrm{MHz}$, major isomer) $\delta \mathrm{l} .66(\mathrm{~s}, 3 \mathrm{H}) .2 .60$ (br s, $1 \mathrm{H}), 3.69$ (s. $\mathrm{lH}$ ). $3.80-3.97(\mathrm{~m}, 4 \mathrm{H}) .6 .70$ (t. $J=2.4 \mathrm{~Hz}, 1 \mathrm{H}), 7.2 \mathrm{l}$ $7.46(\mathrm{~m}, 5 \mathrm{H})$ and ${ }^{l} \mathrm{H} \mathrm{NMR}\left(\mathrm{CDCl}_{\hat{3}}, 300 \mathrm{MHz}\right.$, minor isomer) $\delta 1.71$ (s. $3 \mathrm{H}$ ). 2.54 (br s, lH) 3.78 (s. IH). 3.81 (s, IH). $3.87(\mathrm{~s}, \mathrm{lH}) .3 .91(\mathrm{~d}, J=2.4 \mathrm{~Hz} .2 \mathrm{H}), 6.60(\mathrm{t}, J=2.4 \mathrm{~Hz}$. 1H). $7.21-7.42(\mathrm{~m}, 5 \mathrm{H})$

Compound 5a: $64 \%$ : white solid mp $42-44{ }^{\circ} \mathrm{C}$ : IR (film) 1755. 1687, 1417. 1298. 1199, $1097 \mathrm{~cm}^{-1}:{ }^{1} \mathrm{H} \mathrm{NMR}$ (CDCl${ }_{3}$. $300 \mathrm{MHz}) \delta \mathrm{l} .27$ (t. $J=7.2 \mathrm{~Hz} .3 \mathrm{H}$ ), 1.32 (t. $J=7.2 \mathrm{~Hz} .3 \mathrm{H}$ ). $2.24(\mathrm{~s}, 3 \mathrm{H}) .3 .76(\mathrm{~s} .2 \mathrm{H}), 4.21$ (q. $J=7.2 \mathrm{~Hz} .2 \mathrm{H}), 4.25$ (q. $J$
$=7.2 \mathrm{~Hz} .2 \mathrm{H}$ ). 4.87 (s. 2H). 6.42 (s. IH). $7.12-7.20(\mathrm{~m}, 3 \mathrm{H})$, 7.25-7.30 (m. $2 \mathrm{H}$ ): ${ }^{13} \mathrm{C}$ NMR $\left(\mathrm{CDCl}_{3} .75 \mathrm{MHz}\right) \delta 11.60$, 14.12 . 14.34, 31.24. 51.14, 59.69. 61.30, 119.74, 122.66. $125.84,127.65 .128 .32 .128 .53,128.66,140.81 .162 .08$, 169.27. LCMS $m: 329\left(\mathrm{M}^{-}\right)$.

Compound $\mathbf{5 b}: 47 \%$; colorless oil; IR (film) 1759, 1693. 1444. 1215, 1124. $1099 \mathrm{~cm}^{-1}$; ${ }^{1} \mathrm{H} \mathrm{NMR}\left(\mathrm{CDCl}_{3} .300 \mathrm{MHz}\right) \delta$ 2.23 (s. $3 \mathrm{H}$ ). 3.75 (s. $3 \mathrm{H}$ ). 3.76 (s. $2 \mathrm{H}$ ). 3.79 (s. 3H). 4.87 (s. $2 \mathrm{H}) .6 .43(\mathrm{~s}, \mathrm{lH}), 7.16-7.20(\mathrm{~m}, 3 \mathrm{H}) \cdot 7.24-7.30(\mathrm{~m}, 2 \mathrm{H}){ }^{13} \mathrm{C}$ NMR $\left(\mathrm{CDCl}_{3} .75 \mathrm{MHz}\right) \delta 11.54 .31 .22 .50 .80 .50 .97 .52 .28$. 119.55. 122.77. 125.86, 127.87. 128.33, 128.52. 128.72. $140.68,162.54 .169 .69$

Compound 5c: $56 \%$; colorless oil: IR (film) 1693. 1452 , 1421. 1386, 1297, $1095 \mathrm{~cm}^{-1}$. ${ }^{1} \mathrm{H} \mathrm{NMR}\left(\mathrm{CDCl}_{3} .300 \mathrm{MHz}\right) \delta$ $1.24(\mathrm{t}, J=6.9 \mathrm{~Hz} .3 \mathrm{H}), 2.24$. (s. $3 \mathrm{H}) .3 .76(\mathrm{~s}, 2 \mathrm{H}), 4.19(\mathrm{q} . J$ $=6.9 \mathrm{~Hz} .2 \mathrm{H}$ ). 5.43 (s. $2 \mathrm{H}) .6 .55$ (s. $\mathrm{lH}) .7 .01-7.04(\mathrm{~m}, 2 \mathrm{H})$, 7.14-7.29 (m. 8H): ${ }^{13} \mathrm{C}$ NMR (CDCl $\left.3.75 \mathrm{MHz}\right) \delta 11.69$, $14.28 .31 .21,52.44 .59 .47,119.60,122.29,125.76,126.41$, 127.04, 127.33. 128.28. 128.39, 128.43, 128.59. 138.96, 141.03, 161.86; LCMS $m=333\left(\mathrm{M}^{+}\right)$.

Compound 5d: 41\%; colorless oil; IR (film) 1624, 1495. 1446. $1400,1215.1173 \mathrm{~cm}^{-1}$; ${ }^{1} \mathrm{H} \mathrm{NMR}\left(\mathrm{CDCl}_{3} .300 \mathrm{MHz}\right) \delta$ $1.63(\mathrm{~s}, 3 \mathrm{H}) .3 .73(\mathrm{~s}, 2 \mathrm{H}) .5 .37(\mathrm{~s} .2 \mathrm{H}), 6.68(\mathrm{~s} . \mathrm{HH}), 7.05-$ $7.08(\mathrm{~m}, 2 \mathrm{H}) .7 .16-7.30$ (m, 8H), 7.34-7.40 (m. 2H). 7.44$7.50(\mathrm{~m} . \mathrm{lH}), 7.58-7.6 \mathrm{l}(\mathrm{m}, 2 \mathrm{H}) ;{ }^{12} \mathrm{C} \mathrm{NMR}\left(\mathrm{CDCl}_{3}, 75\right.$ $\mathrm{MHz}) \delta 12.04,31.30 .51 .99,122.72,125.87,126.80,127.28$. $128.16,128.23$. 128.34. 128.39 (2C), 128.45. 128.47. $129.00,129.35 .131 .59 .138 .71,140.73,188.34$; LCMS $m z$ $365\left(\mathrm{M}^{+}\right)$.

Compound 5e: $49 \%$, colorless oil: IR (film) 2208.1493 , 1425. 1390, $1372 \mathrm{~cm}^{-1}$. ${ }^{1} \mathrm{H}$ NMR $\left(\mathrm{CDCl}_{3}, 300 \mathrm{MHz}\right) \delta 2.11$ $(\mathrm{s}, 3 \mathrm{H}), 3.73$ (s. 2H). $4.82(\mathrm{~s}, 2 \mathrm{H}), 6.58(\mathrm{~s}, 1 \mathrm{H}), 7.13-7.16(\mathrm{~m}$, 2H). 7.19-7.33 (m. 3H); ${ }^{13} \mathrm{C}$ NMR $\left(\mathrm{CDCl}_{3}, 75 \mathrm{MHz}\right) \delta$ $10.32 .31 .25,35.66,103.72$. 112.38, 113.39, 124.99. 125.64, $126.42,128.45 .128 .63 .132 .60,139.28$.

Compound 6: $34 \%$; colorless oil: IR (film) 2981.1753 , 1693. 1643, 1251, $1203 \mathrm{~cm}^{-1}$. ${ }^{1} \mathrm{H} \mathrm{NMR}\left(\mathrm{CDCl}_{3} .300 \mathrm{MHz}\right) \delta$ $1.29(\mathrm{t}, J=7.5 \mathrm{~Hz}, 3 \mathrm{H}), 1.38(\mathrm{t} . J=7.5 \mathrm{~Hz}, 3 \mathrm{H}), 2.64(\mathrm{~s}$, $3 \mathrm{H}) .4 .24(\mathrm{q} . J=7.5 \mathrm{~Hz}, 2 \mathrm{H}), 4.32(\mathrm{q} . J=7.5 \mathrm{~Hz}, 2 \mathrm{H}), 4.95$ (s. 2H). 7.06 (s. 1H). 7.43-7.47 (m, 2H). 7.52-7.55 (n. 1H), 7.76 (m. $2 \mathrm{H}):{ }^{12} \mathrm{C}$ NMR $\left(\mathrm{CDCl}_{3}, 75 \mathrm{MHz}\right) \delta 12.55,14.12$, $14.28,51.79,60.47,61.73,121.91,122.65,128.26,129.04$ $131.69,132.49,134.92,140.18,168.24(2 C) .191 .45$ : LCMS $m z 343\left(\mathrm{M}^{-}\right)$

Compound 7: $41 \%$ : pale yellow solid, mp 95-97 ${ }^{\circ} \mathrm{C}$; IR (film) 3303, 2212, $1396 \mathrm{~cm}^{-1} ;{ }^{1} \mathrm{H} \mathrm{NMR}\left(\mathrm{CDCl}_{3} .300 \mathrm{MHz}\right)$ $\delta 2.11$ (s. $3 \mathrm{H}$ ). 3.75 (s. $2 \mathrm{H}$ ). 6.58 (d. $J=3.0 \mathrm{~Hz} . \mathrm{lH}$ ). 7.14 7.22 (m. 3H). 7.26-7.31 (m. 2H), 8.45 (br s, lH): ${ }^{13} \mathrm{C}$ NMR $\left(\mathrm{CDCl}_{3} .75 \mathrm{MHz}\right) \delta 9.96,31.29,100.08 .114 .45,121.97$. $123.62,126.12$. 128.43. 128.46, 130.64, 140.16; LCMS $m z$ $196\left(\mathrm{M}^{+}\right)$.

Acknowledgments. This work was supported by the Korea Research Foundation Grant funded by the Korean Government (MOEHRD, KRF-2006-311-C00384). Spectroscopic data was obtained from the Korea Basic Science Institute. Gwangiu branch. 


\section{References and Notes}

1. For the syntheses and biological activities of pyrrole derivatives. see: (a) Bellina. F; Rossi, R. Tetratredron 2006, 62. 7213-7256. (b) Knight. D. W. Sharland C. M. Sylett 2004. 119-121. (c) Singh, V: Kanojiva, S.: Batra. S. Tetrahedron 2006, 62, 10100 10110 (d) Knight. D. W. Sharland. C. M. Sylet 2003. 22582260. (e) Magnus. N. A.: Staszak. M. A: Udodong. U. E: Wepsiec. J. P. Org. Proc. Res. Dev. 2006. 10. 899-904. (t) Zenl. S.: Harada, K. Chem. Pham. Bull. 1982, 30. 366-369. (g) Chen. Q: Wang. T.: Zhang. Y: Wang Q: Ma. J. Smh. Conmm. 2002 , 32. 1051-1058. (h) Nicolaols. I.: Demopoulos. V. I. J. Med. Chem. 2003. 46. 417-426. (i) Gupton. J. T.: Banner. E. J.: Schart. A. B.: Nonwod. B. K.: Kanters. R. P. F.: Dominey. R. N.: Hempel. J. E.: Kharlamova. A.: Bluhn-Chertudi. I.: Hickenboth. C. R.: Little. B. A.: Sartin. M. D.: Coppock, M. B.: Krumpe. K. E.: Burnham, B. S.: Holt H.: Du K. X.: Keertikar K. M.: Diebes, A.: Ghassemi, S.: Sikorski, J. A. Tetratredron 2006,62. 8243-8255. (j) Cadamuro. S.: Degani. I.: Dughera. S.: Fochi. R: Gatti. A: Piscopo. L. J. Chent. Soc. Perkin Trans. 1 1993. 273-283. (k) Cohnen. E.: Dewald. R. Sinthesis 1987. 566-568. (1) Misra. N. C.: Panda, K.: Ila, H.: Junjappa, H. J. Ong. (Them. 2007. 72 . 12461251.

2. For the examples on the synthesis of prtroles from BavlisHilman adducts. see: (a) Declerck. V.: Ribiere. P.: Martinez, J: Lamaty. F. J. Org. Chent 2004. 69. 8372-8381. (b) Shi. M.: Xu. Y.-M. Eur. J. Org Chem. 2002. 696-701. (c) Rov. A. K.: Pathak. R.: Yadav, G. P.: Maulik, P. R.: Batra, S. Sifthesis 2006. 1021 -
1027.

3. Lee. H. S.: Kiml. T. M.: Kiml. .. N. Tetrohedron Lett. 2007. 48. 4119-4122.

4. When we carried out the reaction in DMF in the presence of $\mathrm{K}_{2} \mathrm{CO}_{3}$ at room temperature, the corresponding intermediates 3 could be isolated in moderate yields.

5. For the synthesis of compound 2d. see: (a) Kawamoto. A.: Wills. M. Tetrahedron: Astmmeny 2000. 11.3257-3261. (b) Guana. A.: Bucelli. I.: Machetti. F.: Menchi. G.: Occhiato. E. G.: Scarpi. D.: Trabocchi. A. Tetrahedron 2002. 58,9865-9870. (c) Deng. B.-L: Demillequand, M; Laurent, M; Touillaux, R.: Belmans, M: Kemps. L: Ceresiat, M: Marchand-Brynaert. J. Tetrahedron 2000. 56. 3209-3217.

6. For the decyanomethylation. see: (a) Katritzky. A. R.: Latif. M: Urogdi. L. J. Chem. Soc. Perkin Trans. I 1990. 667-672. (b) Overman. L. E.: Shin, J. J. Ong. Chem. 1991, 56. $5005-5007$. (c) Yang. T.-K:- Hung. S.-M.: Lee D.-S.; Hong. A.-W.: Cheng, C.C. Tetrahedron Lett. 1989, 30. 4973-4976. (d) Padwa. A.: Chen, Y.Y: Dent. W: Nimmesgern. H. J. Org Chent 1985, 50.4006-4014.

7. For the related PCC oxidations. see: (a) Kim. S. T.: Lee. H. S.: Kim. T. N. Tetrahedron Lett, 2007, 48, 1069-1072. (b) Dauben. W. G:; Michno, D. M. J. Org. Chem. 1977, 42.682-685.

8. For our recent publications on the synthesis of nitrogen-containing five-membered heteroxyclic compounds, see: (a) Lee. K. Y.: Lee. Y. T.: Kiml. J. N. Bull. Korean Chem. Soc. 2007. 28. 143-146. (b) Kim. S. C.: Lee. K. Y.: Gowrisankar. S.: Kim. T. N. Bull. Korean Chem. Soc. 2006. 27. 1133-1139. (c) Lee. H. S.: Kiml. S. J.: Kim. J. N. Bull. Korean Chem. Soc. 2006. 27. 1063-1066 